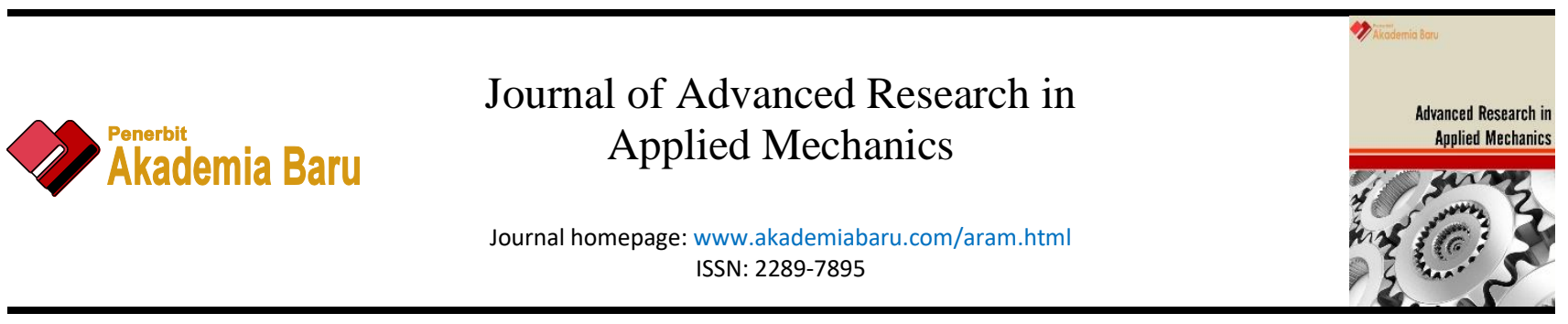

\title{
Design and Fabrication of the Portable Cultural Relic Display Cabinet for Public Exhibition
}

\author{
Quanjin Ma1,2, ${ }^{1}$, Bo Sun ${ }^{2}$, Jian Na², Mohd Ruzaimi Mat Rejab ${ }^{1,2}$, ChinSeik Chong ${ }^{2}$, Weigang Fan², \\ Senyao Tang ${ }^{2}$, Jianxing $\mathrm{Wei}^{2}$
}

1 Structural Performance Materials Engineering (SUPREME) Focus Group, Faculty of Mechanical \& Automotive Engineering Technology, Universiti
Malaysia Pahang, 26600 Pekan, Pahang, Malaysia

2 School of Mechanical Engineering, Ningxia University, 750021 Yinchuan, China

\section{ABSTRACT}

It is known that cultural relics represent the art and aesthetic aspects of ancient history and provides high economic value in the auction market. Cultural relics have many different types, such as jade, porcelain and bronze ware, which are fragile and corrosive but expensive and unique. However, there are two common external conditions do harm to the valuable cultural relic, including humid (e.g. water or flood), high-temperature (e.g. fire) and impact vibration (e.g. earthquake or stealing). Therefore, this paper aims to design and fabricate the portable cultural relic display cabinet, which used to protect the fragile chinaware from irreversibly damaged conditions. The protective method is adopted the lifting system to drop to the display cabinet box under the above conditions. Two damaged conditions have been considered and detected the external conditions, which refers to flame and vibration sensor modules. It was concluded that this device had fast and valid response performance, and the results of experiment reveals it takes approximately $9.8 \mathrm{~s}-10.0 \mathrm{~s}$ to fully lower the porcelain into the main protective body on fire and vibration, such intense conditions. Based on its distinct characteristics above, this device can be widely used in the national museum, the private museum, artwork exhibition, etc.

\section{Keywords:}

Culture relic; display cabinet; lifting system; automation

Copyright @ 2020 PENERBIT AKADEMIA BARU - All rights reserved

\section{Introduction}

In 2017, it was carried out by the museum of the World 2017 that there are more than 55,000 museums in 202 countries, museums enter a golden period of development. For the safety of museums, it does entirely need a particular display device exhibits to the audience, since the cabinet is useful to the museum serves as the media, which exhibits and closely contact with the audience. It is understood that the museum cabinets are an essential part of the museum, they are the last line of defence to protect the safety of cultural relics [1]. Hence, the reasonable and advanced design of museum cabinet is the top prior consideration in display period [2]. However, the reality condition is that relevant researches on museum cabinets are much fewer compared with other fields in the museum, which comes out academic researches on museum cabinets lag far behind the present

\footnotetext{
* Corresponding author.

E-mail address: neromaquanjin@gmail.com
}

https://doi.org/10.37934/aram.71.1.110 
stage in the development of today's museums, intensifying research on museum cabinets becomes more and more urgent [3].

It is known that most cultural relics have become very fragile, and which are vulnerable to the influence of the surrounding environment [4]. Particularly during the regular exhibitions from different countries, if they are not adequate, the cultural relics would easily suffer from irrevocable damages from differential changes, such as temperature, humidity, fire and earthquake in turn. For example, the change of temperature may have universal effects on cultural relics, as shown in Figure 1. The increase in temperature will not only accelerate the chemical reaction rate of cultural relics ageing but also accelerates some physical processes [5]. Such as causing cultural relics to be excessively dry or highly humid, thus causing damage to cultural relics. The phenomenon of thermal expansion and cold contraction caused by temperature changes can also occur on cultural relics, causing deformation and even cracking of cultural relics. Therefore, the temperature standard recommended by various countries for the environment of cultural relics exhibition cabinets is $19^{\circ} \mathrm{C}$ to $24{ }^{\circ} \mathrm{C}$, and the daily fluctuation is less than $1{ }^{\circ} \mathrm{C}[6]$. The suitable method to solve the above problems is to design and manufacture an exhibition cabinet with the temperature detection function.
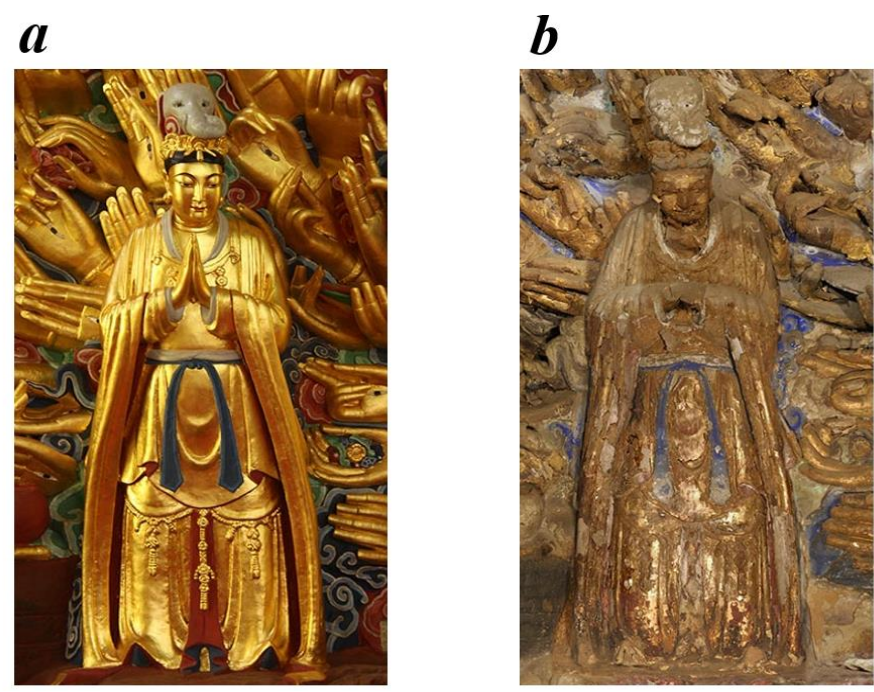

Fig. 1. Damages on part of thousand-armed Avalokitesvara by the change of temperature and humidity: (a) before damage; (b) after damage

Humidity is another essential preservation environmental factor of cultural relics. Oil paintings, textiles and other organic cultural relics are quite sensitive to the moisture in the air, as shown in Figure 2. When the air humidity is high, it absorbs the moisture in the air, and their volume expands accordingly. While air humidity is low, it may shrink. These changes embrittle cultural relics such as paper and textiles, reduce their strength and crack the interior of porcelain [7]. Meanwhile, metal cultural relics are easy to react with water in the air and produce harmful rust, which seriously affects the safety and life of cultural relics [8]. Therefore, A well-sealed display cabinet can provide a relatively stable humidity environment for the preservation of cultural relics [9].

The damage caused by the fire to the cultural relics is incalculable, yet the critical cultural relics are made up of flammable or extremely flammable substances. Such as fire in Notre Dame Cathedral, where a large number of extraordinary works have been lost in disease [10]. Non-combustible or flame-proof material should be employed in the production of exhibition cabinets. As destructive as fire, the earthquakes are extremely destructing movable cultural relics because the losses caused by earthquakes are incalculable. Taking the example of the 2008 Wenchuan earthquake in Sichuan, as 
shown in Figure 3, which illustrates the fragile cultural relic condition before and after this natural disaster. It is reported that 3169 pieces of displayed cultural relics (including 16 pieces of first-class cultural relics) of 216 cultural relics collection associations in Sichuan were different levels of damage [11]. Therefore, the anti-shock factor of display cabinets is a high significant parameter for the protection device [12].

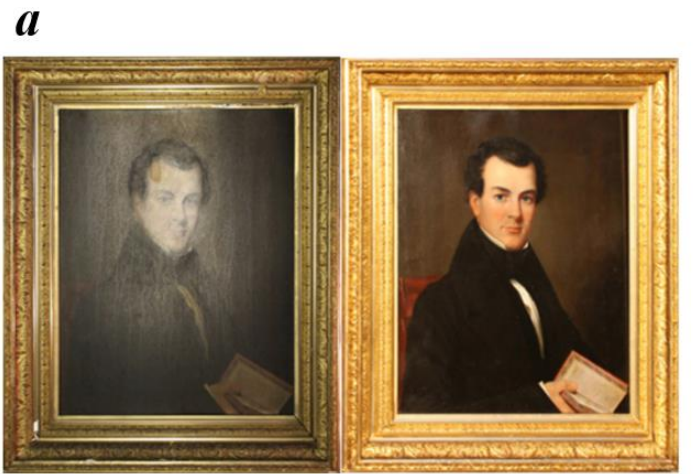

b

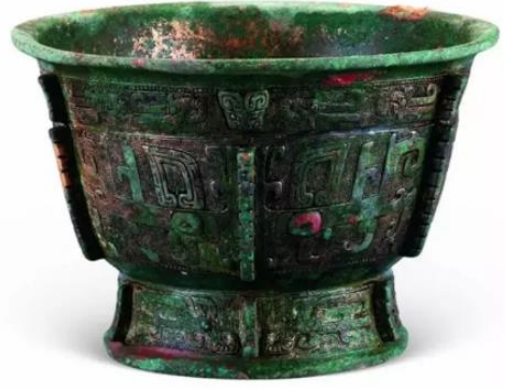

Fig.2. Damages on cultural relics caused by humidity: (a) waterdamaged 19 century oil canvas; (b) Chinese bronze produces harmful rust

$\boldsymbol{a}$

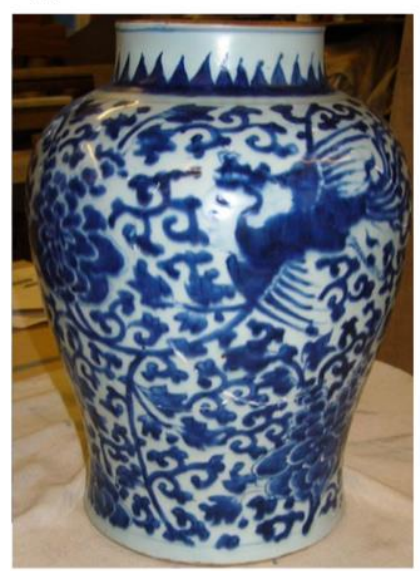

$b$

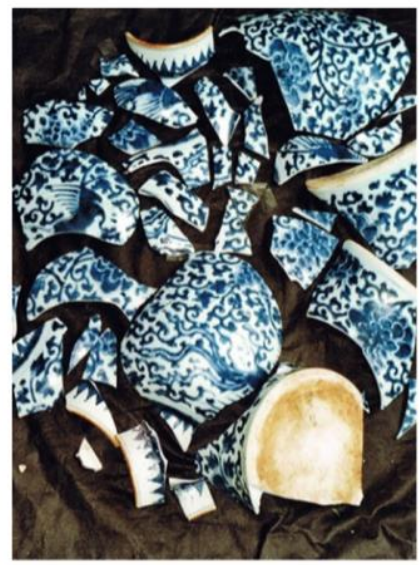

Fig.3. Damage on Ming vase caused by earthquake: (a) before the earthquake; (b) after the earthquake

With growing amounts of museum opening to the public, more and more citizens get chances to be exposed to many precious collections, whereas the safety protection of cultural relics also is emphasised [13]. In the continuous development of museum display cabinets, safety factor has primarily been taken into consideration. For example, armoured glass is applied to the display cabinets, which plays an important role in preventing cultural relics from the external environment. However, due to limitation of technology at that time, the security protection of cabinets in the museum try to concentrate on the external environment and hardly pay considerable attention to the aspects of anti-theft, fire prevention and earthquake prevention of itself [14]. It is shown that resulting in the cabinets becomes the weakest link in the security protection of the whole museum. In addition, there are some problems in undeveloped cities, such as limited storage location, small building area and outdated equipment, which can hardly provide normal storage conditions for many 
cultural relics [15]. And with the change of environmental protection, some historical, artistic and scientific information of those cultural relics may be gradually lost [16]. Finally, it must be mentioned that a lot of visitors could barely have awareness of cultural relics protection. So if the cultural relics protection measures are not perfect, visitors may accidentally damage the cultural relics during the visit [17]. Therefore, an innovative display design can not only effectively protect cultural relics, but also shorten the distance between visitors and cultural relics so as to make the museum attract a large number of visitors, and achieve the best display effect [18].

This paper is aimed to design and fabricate the automatic protective cabinet to prevent the fragile cultural relic artworks from two damaged conditions, including fire (or high temperature), water (or humidity), earthquake or stealing condition (or vibration). The method is used the lifting component to automatically protect the fragile artworks in the exhibition box under the above conditions. Meanwhile, this portable device is evaluated based on its two possible damaged conditions, which provides quick and valid respond performance.

\section{Method}

\subsection{Material Preparation}

The acrylic plate is used to fabricate both the protection container and the transparent display cabinet. Inside, the inner framework and the transmission structure are manufactured using aluminum profile. This device is referred to several portable devices with open-source software and laboratory-scale size, which provides the design and fabrication concepts [19-22]. For control system components, it is mainly used Arduino Uno as the main micro-controller, and two types of sensor modules are selected and aimed to detect the surrounding environment. Furthermore, the NEMA stepper motor is used to provide the rotation method for lifting system, and the scissor lift is employed to match with the prior motor to drive the cultural relics up and down when the sensors are activated. The switch button is placed at the bottom part of this device to start and shut down its action. The relevant components of this device are summarised in Table 1, which includes vital specifications and several parameters.

Table 1

Specifications of several components used in this study

\begin{tabular}{ll}
\hline Components & Specification \\
\hline Scissor lift & $20 \times 20 \times 15 \mathrm{~cm}$ \\
Arduino Uno microcontroller & Operating voltage: $5 \mathrm{~V} /$ Digital I/O pins: 14 \\
5 way flame sensor module & Voltage: $3.3-9 \mathrm{~V} /$ Detecting range: > 120 degree \\
SW-420 vibration sensor module & Operating voltage: $3.3-5 \mathrm{~V}$ \\
Rain sensor raindrop module & Module supply: $12 \mathrm{~V} / 5-12 \mathrm{~mA}$ quiescent current \\
SY57STH56 NEMA23 stepper motor & Rated current: $4.2 \mathrm{~A} /$ Holding torque: $3 \mathrm{~N} \cdot \mathrm{m}$ \\
\hline
\end{tabular}

\subsection{Fabrication Procedure}

\subsubsection{Design concept}

There are mainly two ways to store the movable cultural relics in the museums, which are display and storage functions. Taking display as a demonstration, while most of the movable cultural relics are directly floating on the ground or floor, or fixed on the tabletop of the exhibition cabinet or the 
tabletop of the base [9]. Regarding this condition, it is put forward the shape of the automatic protective cabinet should be forged as a standard exhibition cabinet, which could be quickly adapted to museums. The initial 3D structure is designed using Solidworks software to present its design concept in details, as shown in Figure 4. The device has two primary functions, the first function is to display collections, and secondly, it is to protect the cultural relics in different museums or exhibitions. It is divided into an upper transparent display cabinet, framework section and interior transmission section.

To ensure the significant effect of the exhibition and to protect cultural relics simultaneously, an airtight glass container is utilised to exhibit the cultural relics. This sort of glass display cabinet with excellent sealing performance can not only provide a relatively stable humidity environment for cultural relics but also can isolate harmful gases from the outside environment. Meanwhile, the framework is designed to place the transparent cabinet where contains the cultural relics, the transmission system, and the control system are fixed inside as well. The transmission section is designed to offer stable power to the transparent display cabinet extending and retracting from the framework when the emergency disasters occurs, like fire, earthquake, etc.

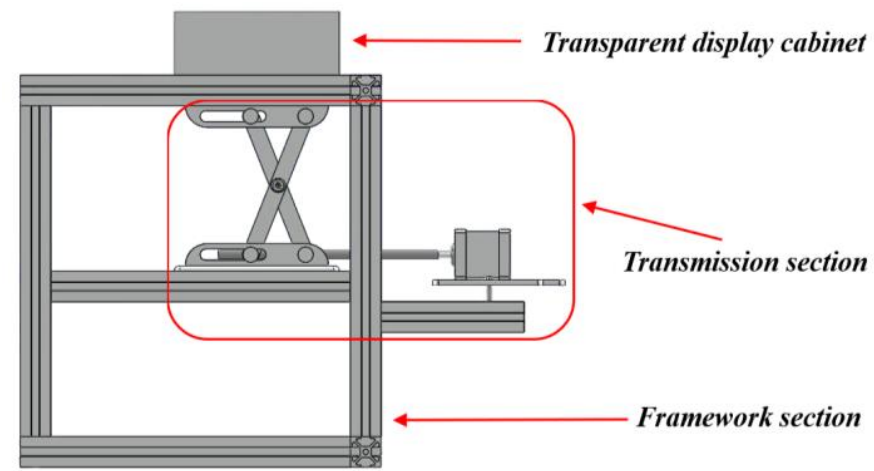

Fig.4. The initial design concept of this portable cultural relic display cabinet

The finalised design structure of this device is presented in Figure 5(a), which includes the transparent display cabinet, framework and the transmission section. A study on earthquake protection of cultural relics in museums demonstrates when an earthquake occurs, the most likely danger to cultural relics are collision with cabinet, falling onto the ground, and motion of the pedestal [23]. To guarantee the anti-shock function of the cabinet itself, the frame is made out of materials with high anti-collision strength and its structure are shaped into cube. When massive objects hit the device, the high anti-collision frame is good at resisting the impact, to preserve the cabinet itself and adequately protect the cultural relics stored inside. As a device that can provide automatic protection service, its transmission principle is to use the stepper motor drive the lead screw that matched with a scissor lift to move forward and backward, and furtherly force the scissor lift open and close to get the purpose that let the transparent display cabinet extend and retract quickly.

Besides, the purpose of converting the rotation to the movement of the lead screw, a pair of pulleys is fixed on the bottom of the bracket where the stepper motor locates. When the lead screw starts to rotate, the pully in the chute is going to move to make the lead screw forward and backwards simultaneously. The modern design standards in museum point out the materials used in the showcase, such as backboard, exhibition stand, and bracket, should be made of the flame-proof materials. These materials hold excellent physical properties, which enable keep the showcase moisture-proof, mildew-proof, and strong enough to withstand the earthquake [24]. Therefore, this device is wrapped up outside by the fire-proof materials. Furthermore, physical shock absorption is 
achieved by placing a soft elastic substance in a confined space, the cross-section view of the device is shown in Figure 5(b). Figure 6 presents the wiring diagram of the portable cultural relic display cabinet, which involves Arduino Uno, stepper motor, motor driver, motor power supply connector, 5 way flame sensor module, vibration sensor module, power supply connector and push button. The sensitivity of two types of sensors could be adjusted via its potentiometer, which depends on its external conditions.
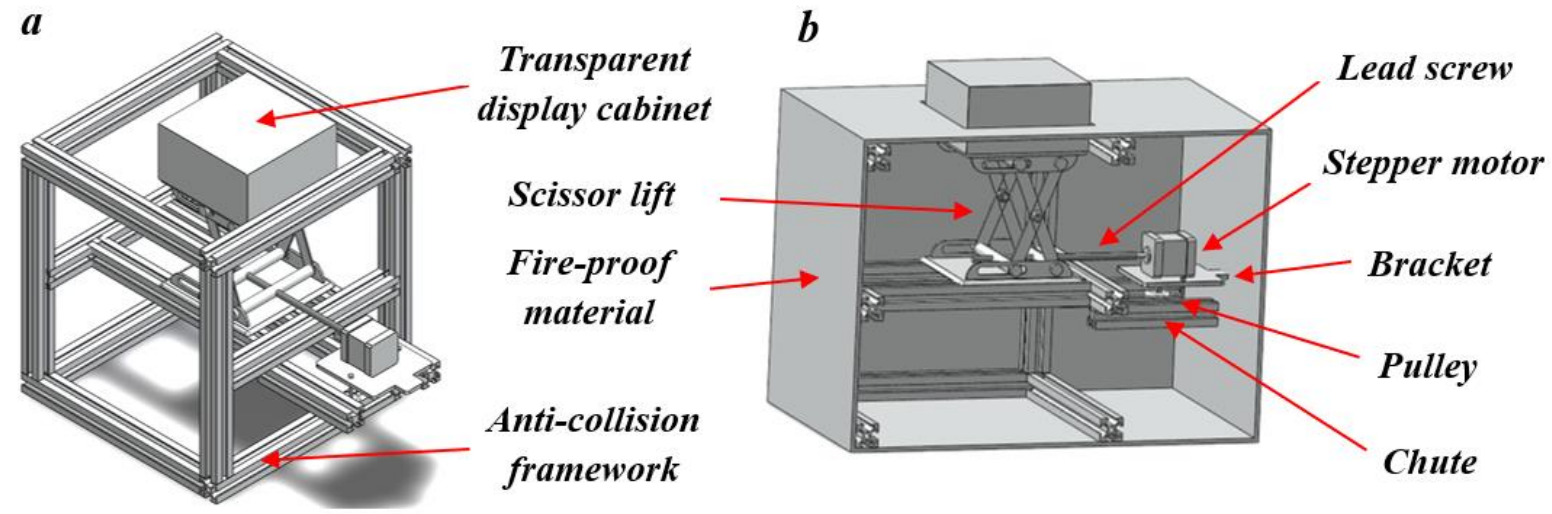

Fig. 5. The 3D prototype of this portable cultural relic display cabinet: (a) isometric view; (b) cross-section view

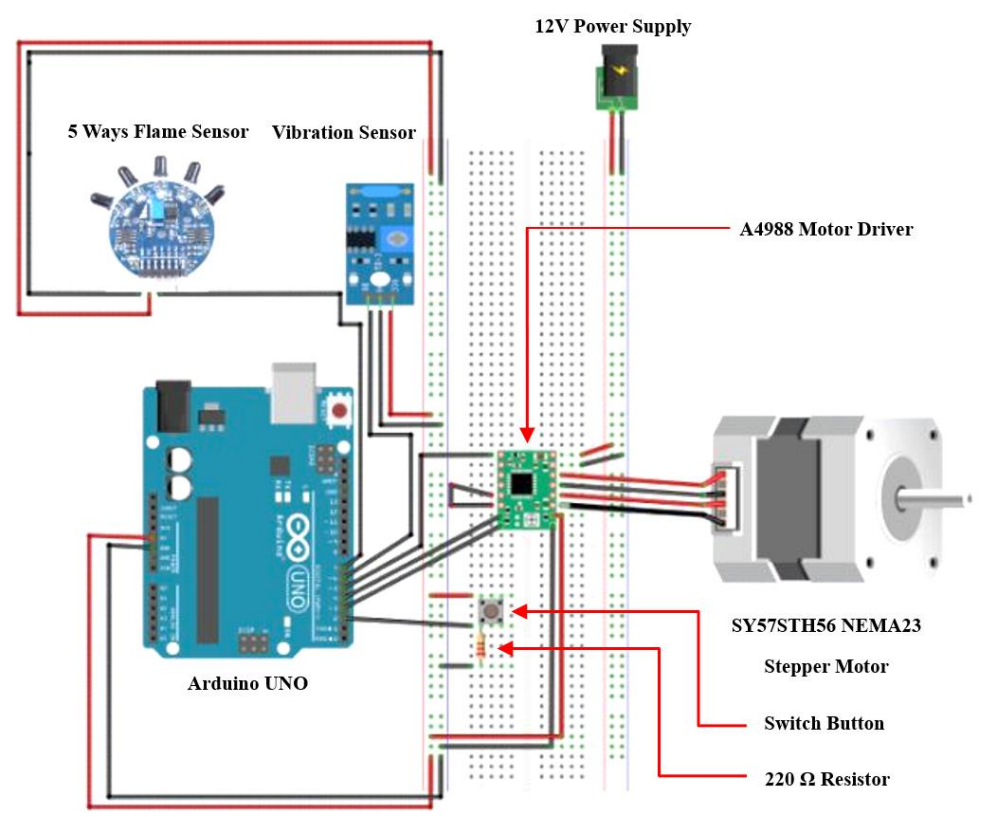

Fig. 6. Wiring diagram of the main components used in this study

\subsubsection{Fabrication procedure}

Flowchart of the portable cultural relic display cabinet is illustrated in Figure 7. Based on its fabrication procedure, the framework is initially fabricated. It consists of aluminum profile that is 500 $\times 330 \times 350 \mathrm{~cm}$ (Dimension: length $\times$ width $\times$ height), and each aluminum profile connects by mounting bracket, nuts and screws. The transmission section is placed inside the framework, which is made up of a scissor lift and a stepper motor. Both scissor lift and stepper motor are fixed in the 
position of the bracket connected to the framework. The third assembly part is the transparent display cabinet, which is installed on the top of the scissor lift, and the glass is utilised to join together with glue to be an airtight transparent display cabinet. The control system is placed in the control system zone of the framework, and the rest of the space of the device is filled with fire-proof materials. In the end, the whole device is wrapped up with the acrylic plate, and the switch button is ultimately placed on the lateral side of the device to start and shut down. The calibration procedure is performed to test the response performance according to its design function. There are several parameters should be finalised, such as limit switch position, transmission shaft centre distance, and sensitivity value.

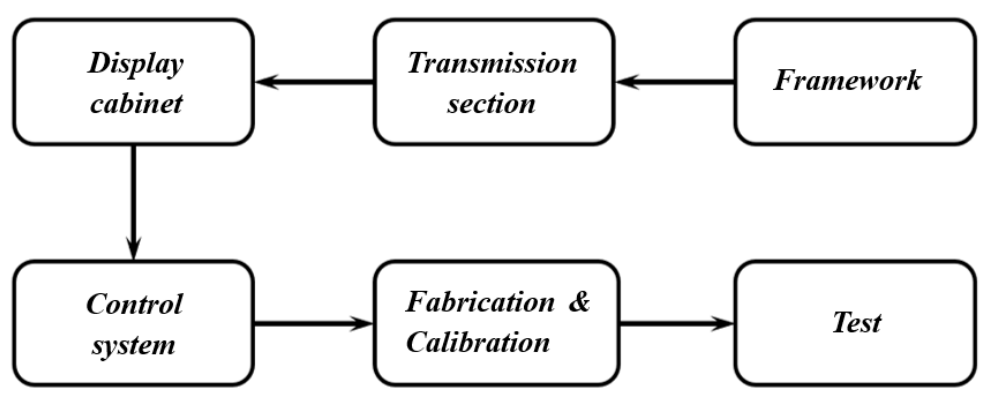

Fig. 7. Flowchart of the portable cultural relic display cabinet

This device is initially fabricated using Solidwork software, which refers to structural design, dimension, concept and component arrangement. Figure 8 (a) presents the 3D prototype front view of the portable cultural relic display cabinet, which is modified according to its initial design concept. It is concluded that the portable cultural relic display cabinet successfully designed and fabricated in term of the optimal engineering drawing. It is designed to automatically raise and lower the cultural relics when detecting the external condition changing to achieve the protective method. Glass display cabinet is adopted to protect the inner cultural relics from outer environment, and the acrylic plate is used to enclose the whole frame of the device with golden colour, as shown in Figure 8 (b).
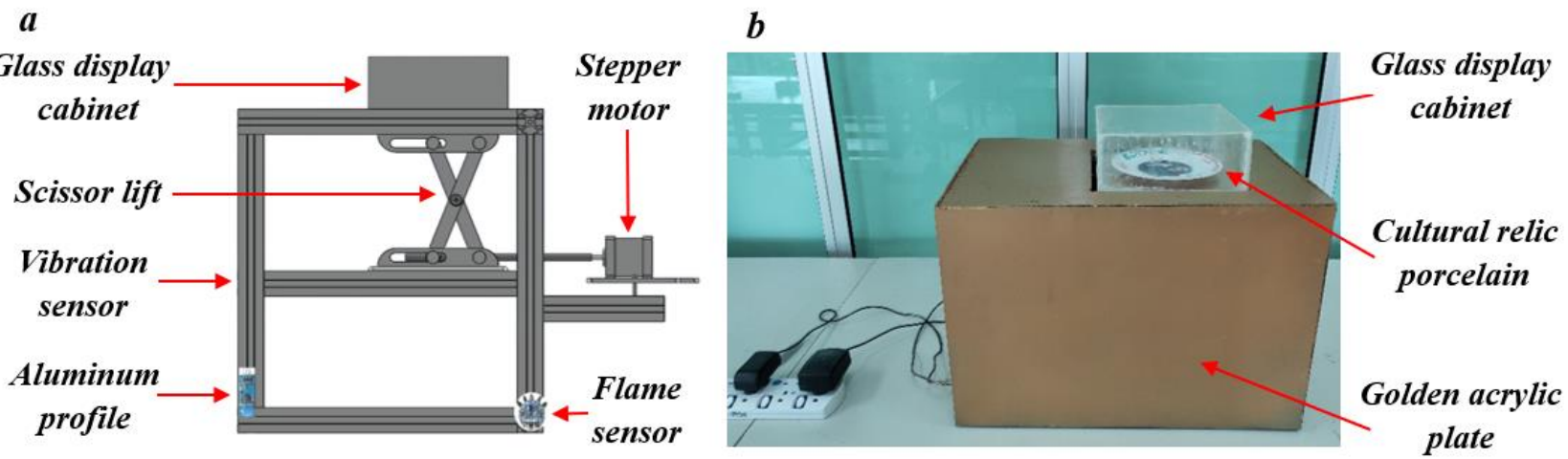

Fig. 8. Schematic structure of the portable cultural relics protective device: (a) 3D prototype structure;

(b) realised prototype device

\section{Results and Discussion}

To evaluate its protective function, response performance of the portable cultural relics protective device is studied and tested, which involves fire and vibration conditions. The results of 
experiments show that the protective device is automatedly extended and retract when the relevant sensors detect the two emergency conditions. For water condition, it is proved the airtight glass display cabinet is good at preventing the inner porcelain from the water drop and keep the room environment be in constant temperature and humidity when pouring water onto the cabinet, as shown in Figure 9. Fire condition is shown in Figure 10, it is tested using the lighter to close to the 5channel infrared flame sensor with above 120 degrees detection range. For vibration condition, it is detected by knocking on the device using hand, which is closed to simulate the earthquake or stealing conditions, as shown in Figure 11. It is conducted that it takes approximately $9.8 \mathrm{~s}-10.0 \mathrm{~s}$ to fully lower the porcelain into the main protective body on two different damage conditions.

$a$

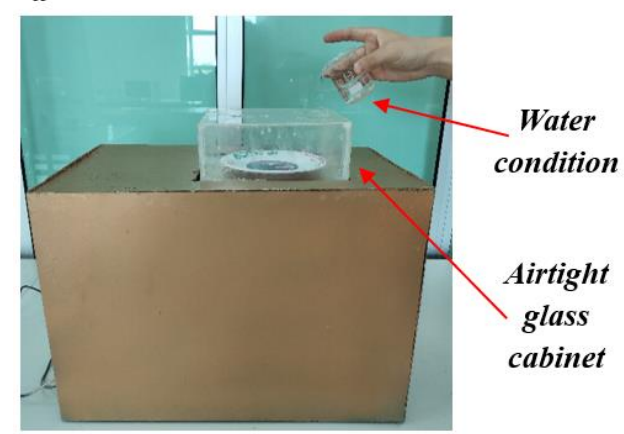

$b$

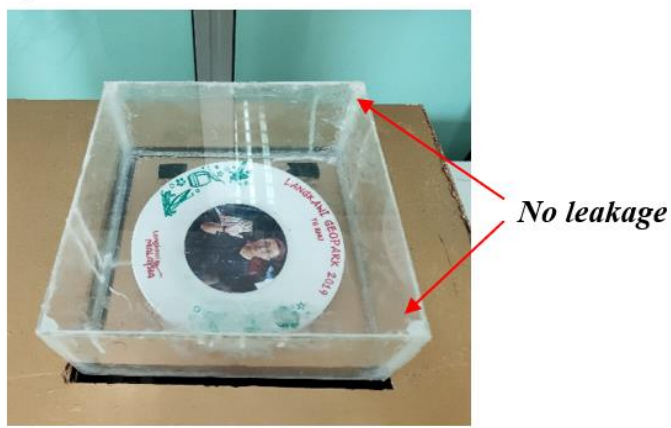

Fig.9. Response performance under water condition: (a) before; (b); after

$a$

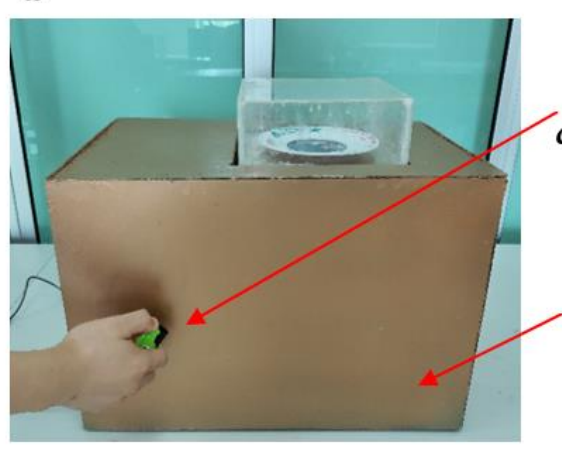

b

$\begin{gathered}\text { Fire } \\ \text { ondition }\end{gathered}$
5 way
flame
sensor
module

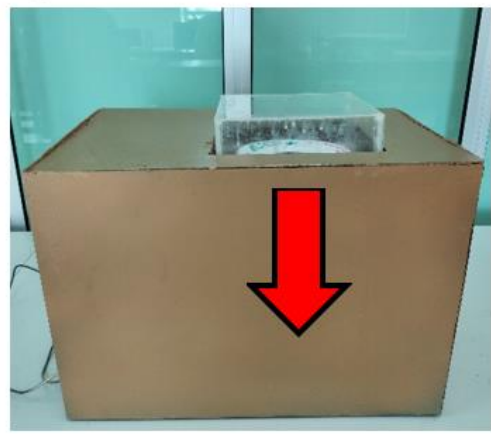

Fig.10. Response performance under fire condition: (a) before; (b) after

$a$

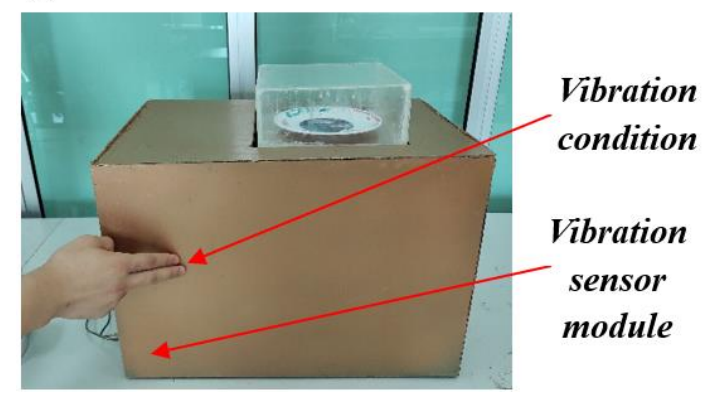

b

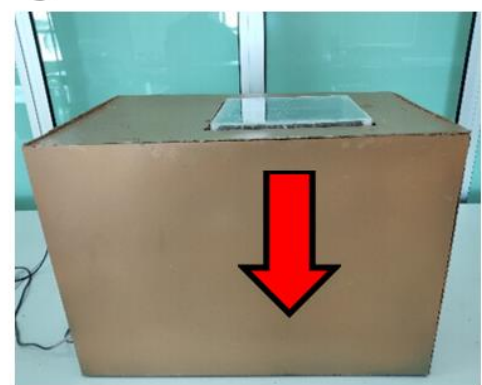

Fig. 11. Response performance under vibration condition: (a) before; (b) after

Figure 12 presents the manual control method using the button of the switch, which is used to relieve the protective method and another purpose. According to date we collected, it takes $9.8 \mathrm{~s}$ $10.0 \mathrm{~s}$ to retract, and around $19.7 \mathrm{~s}-20.0 \mathrm{~s}$ to expend to the display position. The porcelain is manually hidden by security personnel when the art exhibition is closed, which effectively reduce stealing chances. 


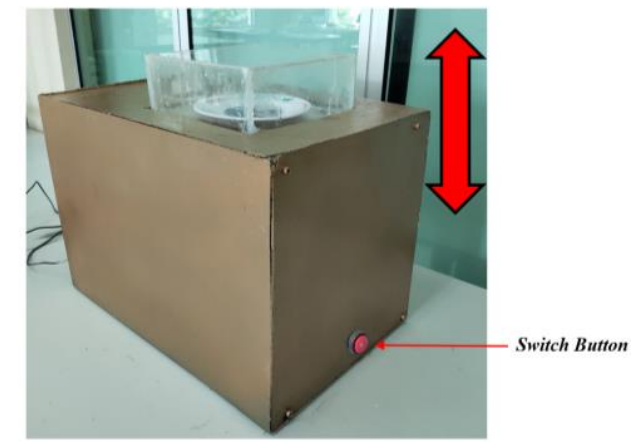

Fig. 12. Manual control button for response performance

\section{Conclusions}

The portable cultural relics protective device is successfully designed, fabricated and tested according to water, fire and vibration conditions, which is used to simulate possible damaged or stealing conditions. Based on its tested results, it is concluded that this device offers the strong response performance, which is automatedly lifting the cultural relics up around $20 \mathrm{~s}$ and letting it lower within the span of $10 \mathrm{~s}$. The button is used to manually control the cultural relics movements such as extending and retracting and pause at a certain position. The cultural relic is placed inside the glass display cabinet, which is simply to take out and replace another collection. Also, this device provides sufficient procedure to protect the cultural relics, which offers the new potential method to reduce the irreversible damaged of valuable collection.

\section{Acknowledgement}

The authors are grateful to the Human Engineering Focus Group (HEG) and Structural Performance Materials Engineering Focus Group (SUPREME) to provide the components and equipment. Meanwhile, this research is funded by Faculty of Mechanical \& Automotive Engineering Technology, Universiti Malaysia Pahang: PGRS180319.

\section{References}

[1] Yi, W. A. N. G. "On Semi-Colonial Museums and Semi-Colonial Image: Focused on the Museum of Royal Asiatic Society at Shanghai [J]." Southeast Culture 2 (2013).

[2] Berndt, Guido M., and Roland Steinacher. "Minting in Vandal North Africa: coins of the Vandal period in the Coin Cabinet of Vienna's Kunsthistorisches Museum." Early Medieval Europe 16, no. 3 (2008): 252-298. https://doi.org/10.1111/j.1468-0254.2008.00231.x

[3] Pearce, Susan. Museums, objects, and collections: A cultural study. Smithsonian Institution, 2017.

[4] McLaughlin, Paul, and Thomas Dietz. "Structure, agency and environment: Toward an integrated perspective on vulnerability." Global environmental change 18, no. 1 (2008): 99-111. https://doi.org/10.1016/i.gloenvcha.2007.05.003

[5] Fang Liujing, "The Influence of Climate Factors on the Atmospheric Corrosion of the Simulated Bronze Cultural Relics", Journal of East China University of Science and Technology (Natural Science Edition) 4, (2015): 489-494.

[6] Yulin, CHEN Yuansheng XIE. "Studies on museum environmental standards." Sciences of Conservation and Archaeology (2002): S1.

[7] Karp, Ivan. EXHIBITING CULTURES PB. Smithsonian, 1991.

[8] Jingsheng, Li. "A discussion on the restoration and repair of cultural relics and their practices [J]." China Cultural Heritage Scientific Research 3 (2012).

[9] Wang Yali, "Discussion on the Environmental Control of Museum", Wind Technology, 13 (2011): 245-245.

[10] Ferreira, Tiago Miguel. "Notre Dame Cathedral: Another case in a growing list of heritage landmarks destroyed by fire." Fire 2, no. 2 (2019): 20. https://doi.org/10.3390/fire2020020 
[11] Qian, Z. H. O. U., Y. A. N. Weiming, Z. H. O. U. Xiyuan, and Zhang Bo. "Aseismic behavior of Chinese ancient tenonmortise joint." Journal of Vibration, Measurement \& Diagnosis 31, no. 6 (2011): 679-684.

[12] Du, Qiao-Hui, Cheng Peng, and Hong Zhang. "Polydatin: a review of pharmacology and pharmacokinetics." Pharmaceutical biology 51, no. 11 (2013): 1347-1354. https://doi.org/10.3109/13880209.2013.792849

[13] Newell, Phillip. "The PRC's Law for the Protection of Cultural Relics." Art Antiquity \& L. 13 (2008): 1

[14] Teygeler, René, Gerrit de Bruin, Bihanne Wassink, and Bert Van Zanen. "Preservation of archives in tropical climates: an annotated bibliography." ICA, 2001.

[15] Shi Yilong, A Brief Talk on Several Issues in the Protection of National Traditional Culture. 2005.

[16] Zhang Lei, et al., Talking about the current situation and thinking of cultural relics protection in basic museums. Cultural Relics Restoration and Research, 2016(0): p. 127.

[17] Ma Huili, et al., Multi-scale analysis of the spatial distribution characteristics of Chinese museums. Human geography, 2017. 32(6): p. 87-94.

[18] Guan Xinjue, How to innovate the design method of cultural relics museum display. Scientific Chinese (2017): 132.

[19] Quanjin, Ma, M. R. M. Rejab, M. S. Idris, B. Bachtiar, J. P. Siregar, and M. N. Harith. "Design and optimize of 3-axis filament winding machine." In IOP Conference Series: Materials Science and Engineering, vol. 257, no. 1, p. 012039. IOP Publishing, 2017. https://doi.org/10.1088/1757-899X/257/1/012039

[20] Quanjin, Ma, M. R. M. Rejab, Nallapaneni Manoj Kumar, and M. S. Idris. "Experimental assessment of the 3-axis filament winding machine performance." Results in Engineering 2 (2019): 100017. https://doi.org/10.1016/j.rineng.2019.100017

[21] Quanjin, Ma, Zhang Zhenggang, M. R. M. Rejab, M. S. Idris, Bo Zhang, and M. H. Abdullah. "Design and fabrication of the portable artwork preservative device (PAPD)." Journal of Modern Manufacturing Systems and Technology 3 (2019): 31-38.

[22] Quanjin, Ma, M. Rejab, I. Sahat, M. Amiruddin, D. Bachtiar, J. P. Siregar, and M. I. Ibrahim. "Design of portable 3axis filament winding machine with inexpensive control system." J Mech Eng Sci 12 (2018): 3479-3493. https://doi.org/10.1088/1757-899X/257/1/012039

[23] Zhongliang, Wang. "A Study on Earthquake Protection of Cultural Relics in Museums." Earthquake Resistant Engineering 1 (2004).

[24] Zhang Le, Research on the interior design of museum display cabinets. Journal of Nanjing University of the Arts: Fine Arts and Design Edition, 2015(5): 201-205. 